\title{
EXISTENCE AND UNIQUENESS FOR THE THREE-DIMENSIONAL THERMOELASTICITY SYSTEM IN SHAPE MEMORY PROBLEMS
}

\author{
IRENA PAWŁOW and ANTONI ŻOCHOWSKI \\ Systems Research Institute of the Polish Academy of Sciences \\ ul. Newelska 6, 01-447 Warszawa, Poland \\ E-mail: pawlow@ibspan.waw.pl,zochowsk@ibspan.waw.pl
}

\begin{abstract}
A thermodynamically consistent model of shape memory alloys in three dimensions is studied. The thermoelasticity system, based on the strain tensor, its gradient and the absolute temperature, generalizes the well-known one-dimensional Falk model. Under simplifying structural assumptions we prove global in time existence and uniqueness of the solution.
\end{abstract}

1. Introduction. In the paper we consider mathematical properties of the threedimensional thermoelasticity system representing the behaviour of the body made of shape memory alloy (SMA). This system is obtained from thermodynamical relations [8] and generalizes the one-dimensional Falk model [3].

The equations to be considered are given by the following Problem (P):

$$
\begin{aligned}
\mathbf{u}_{t t}-\nu \mathbf{Q} \mathbf{u}_{t}+\frac{\kappa}{4} \mathbf{Q Q u} & =\boldsymbol{\nabla} \cdot F_{/ \boldsymbol{\epsilon}}(\boldsymbol{\epsilon}, \theta)+\mathbf{b} \\
c(\boldsymbol{\epsilon}, \theta) \theta_{t}-k \Delta \theta & =\theta F_{/ \theta \boldsymbol{\epsilon}}(\boldsymbol{\epsilon}, \theta): \boldsymbol{\epsilon}_{t}+\nu\left(\mathbf{A} \boldsymbol{\epsilon}_{t}\right): \boldsymbol{\epsilon}_{t}+g
\end{aligned}
$$

in $Q_{T}=(0, T) \times \Omega$, where

$$
c(\boldsymbol{\epsilon}, \theta)=c_{v}-\theta F_{/ \theta \theta}(\boldsymbol{\epsilon}, \theta)
$$

with boundary conditions

$$
\begin{gathered}
\mathbf{u}=0, \quad \mathbf{Q u}=0, \\
\nabla \theta \cdot \mathbf{n}=0 \quad \text { on } S_{T}=(0, T) \times \partial \Omega,
\end{gathered}
$$

2000 Mathematics Subject Classification: 35Q72, 35K50, 35K60, 74B20.

The paper is in final form and no version of it will be published elsewhere. 
and initial conditions

$$
\begin{gathered}
\mathbf{u}(0, \mathbf{x})=\mathbf{u}_{0}(\mathbf{x}), \quad \mathbf{u}_{t}(0, \mathbf{x})=\mathbf{u}_{1}(\mathbf{x}), \\
\theta(0, \mathbf{x})=\theta_{0}(\mathbf{x}) \quad \text { in } \Omega
\end{gathered}
$$

Here $\Omega \subset \mathbb{R}^{n}, n=2$ or 3 , is a bounded domain with a smooth boundary $\partial \Omega$, u denotes the displacement vector, $\boldsymbol{\epsilon}=\left(\epsilon_{i j}\right)$ with $\epsilon_{i j}(\mathbf{u})=\frac{1}{2}\left(u_{i / j}+u_{j / i}\right)$ is the linearized strain tensor, $\boldsymbol{\epsilon}_{t}=\boldsymbol{\epsilon}\left(\mathbf{u}_{t}\right)$ is the strain rate tensor, and $\theta>0$ is the absolute temperature. The other quantities in (1.1)-(1.2) have the following meaning: $F(\boldsymbol{\epsilon}, \theta)$ - elastic energy, $c(\boldsymbol{\epsilon}, \theta)$ - specific heat coefficient, $\mathbf{b}, g$ - external body forces and heat sources, $c_{v}, k, \nu$, $\kappa$ - positive numbers representing respectively thermal specific heat, heat conductivity, viscosity and interfacial energy coefficient.

The elastic energy $F(\epsilon, \theta)$ has a multiple-well form as a function of the strain tensor $\epsilon$ (order parameter), with the shape changing qualitatively with temperature $\theta$. These changes correspond to the fact that the austenitic phase is the global minimizer above a critical temperature, both the austenitic phase and the martensitic variants have equal energy density at the critical temperature, and the martensitic variants are global minimizers below the critical temperature. As a representative model of $F(\epsilon, \theta)$ we use the Falk-Konopka elastic energy [4] in the form of sixth order polynomial of variables $\epsilon_{i j}$ expressed in terms of crystallographical invariants, generalizing the well-known 1-D expression [3],

$$
F(\epsilon, \theta)=\alpha_{1}\left(\theta-\theta_{c}\right) \epsilon^{2}-\alpha_{2} \epsilon^{4}+\alpha_{3} \epsilon^{6}
$$

where $\alpha_{i}>0$ are constant parameters and $\theta_{c}>0$ is a critical temperature.

Further, $\mathbf{A}=\left(A_{i j k l}\right)$ with

$$
A_{i j k l}=\lambda \delta_{i j} \delta_{k l}+\mu\left(\delta_{i k} \delta_{j l}+\delta_{i l} \delta_{j k}\right),
$$

is the fourth order tensor representing linear isotropic Hooke's law

$$
\boldsymbol{\epsilon}(\mathbf{u}) \mapsto \mathbf{A} \boldsymbol{\epsilon}(\mathbf{u})=\lambda \operatorname{trace} \boldsymbol{\epsilon}(\mathbf{u}) \mathbf{I}+2 \mu \boldsymbol{\epsilon}(\mathbf{u}),
$$

where $\lambda, \mu$ are Lamé constants and $\mathbf{I}=\left(\delta_{i j}\right)$ is the unit matrix. We assume $\mathbf{A}$ is coercive and bounded,

$$
a_{\star}|\boldsymbol{\epsilon}|^{2} \leq(\mathbf{A} \boldsymbol{\epsilon}): \boldsymbol{\epsilon} \leq a^{\star}|\boldsymbol{\epsilon}|^{2}
$$

where

$$
a_{\star}=\min [n \lambda+2 \mu, 2 \mu], \quad a^{\star}=\max [n \lambda+2 \mu, 2 \mu] .
$$

Moreover, $\mathbf{Q}$ stands for the second order differential operator of linearized elasticity defined by

$$
\mathbf{u} \mapsto \mathbf{Q u}=\boldsymbol{\nabla} \cdot(\mathbf{A}(\boldsymbol{\epsilon}))=\mu \Delta \mathbf{u}+(\lambda+\mu) \boldsymbol{\nabla}(\boldsymbol{\nabla} \cdot \mathbf{u}) .
$$

The term $\nu \mathbf{Q} \mathbf{u}_{t}$ in the elasticity equation corresponds to the mechanical viscosity governed by Hooke's law and the related term $\nu\left(\mathbf{A} \epsilon_{t}\right): \boldsymbol{\epsilon}_{t}$ in the temperature equation represents heat production due to viscous dissipation.

The fourth order term $\frac{\kappa}{4} \mathbf{Q Q u}$ corresponds to interaction effects on phase interfaces which are expressed by the strain gradient contribution $\frac{\kappa}{8}|\mathbf{Q u}|^{2}$ to the free energy density

$$
f(\boldsymbol{\epsilon}, \nabla \boldsymbol{\epsilon}, \theta)=-c_{v} \theta \log \theta+F(\boldsymbol{\epsilon}(\mathbf{u}), \theta)+\frac{\kappa}{8}|\mathbf{Q u}|^{2} .
$$


There exist several three-dimensional continuum models describing describing thermomechanical evolution of SMA. One of them, the well-known Frémond model [5], is based on the strain tensor, the volumetric proportions of austenite and martensite, and the absolute temperature as state variables. The interfacial structure is there accounted for by the gradient of the strain tensor trace. The well-posedness of such a model has been studied by several authors (see [1] for references).

Our contribution consists in proving global in time existence and uniqueness results for the fourth order viscoelasticity system (1.1) coupled with the temperature equation (1.2). We prove that the system is well posed for $(\mathbf{u}, \theta)$ in the space $\mathbf{W}_{p}^{4,2}\left(Q_{T}\right) \times W_{p}^{2,1}\left(Q_{T}\right)$ with $p \geq n+2$. In such a case the strain tensor $\epsilon$, its gradient $\nabla \epsilon$ and the temperature $\theta$ are continuous functions in time-space cylinder $Q_{T}$.

The approach used in this paper relies on the parabolic decomposition of the elastic part (1.1) and the subsequent application of the Leray-Schauder fixed point theorem to the decomposed system coupled with the temperature equation.

In the present paper we shall concentrate on the central part of the existence proof, namely establishing a priori bounds for the solution by using the recursive improvement technique and parabolic decomposition. This required proving several auxiliary results, for which we refer the reader to [9].

Notation. Let $\Omega_{t}=\{t\} \times \Omega, Q_{t}=(0, t) \times \Omega, S_{t}=(0, t) \times \partial \Omega$, and $\mathbf{n}$ stand for the unit outward normal to $\partial \Omega$. Moreover, $f_{/ i}=\partial f / \partial x_{i}, f_{t}=\partial f / \partial t$.

We use the Sobolev spaces notation of [6] and bold letters for the vector- and tensorvalued mappings.

The summation convention over repeated indices is assumed and the following notation for vectors $\mathbf{a}=\left(a_{i}\right), \mathbf{b}=\left(b_{i}\right)$ and tensors $\mathbf{B}=\left(B_{i j}\right), \tilde{\mathbf{B}}=\left(\tilde{B}_{i j}\right)$ :

$$
\mathbf{a} \cdot \mathbf{b}=a_{i} b_{i}, \quad \mathbf{B}: \tilde{\mathbf{B}}=B_{i j} \tilde{B}_{i j}, \quad|\mathbf{B}|=\left(B_{i j} B_{i j}\right)^{1 / 2} .
$$

The symbols $\nabla, \nabla \cdot$ denote the gradient and the divergence operators. For the divergence of a tensor field $\boldsymbol{\epsilon}(\mathbf{x})=\left(\epsilon_{i j}(\mathbf{x})\right.$ the convention of the contraction over the last index is used, i.e.,

$$
\boldsymbol{\nabla} \cdot \boldsymbol{\epsilon}(\mathbf{x})=\left(\epsilon_{i j / j}(\mathbf{x})\right) .
$$

Throughout the paper $\Lambda$ denotes a generic constant, different in various instances. In general, $\Lambda$ can depend on the data of the problem, domain $\Omega$ and time $T$.

Acknowledgements. The authors would like to thank Professor Wojciech Zajączkowski for detailed discussions.

2. Assumptions and main results. Below we list our assumptions grouped into several categories.

(D) Domain $\Omega \subset \mathbb{R}^{n}, n=2,3$, with the boundary $\partial \Omega$ of the class $C^{3}$.

(LP) Linear part: the coefficients of the operator $\mathrm{Q}$ satisfy conditions

$$
\mu>0, \quad n \lambda+2 \mu>0 .
$$


They ensure the coercivity of the algebraic operator $\mathbf{A}$ in (1.8) and also imply the strong ellipticity of the operator $\mathbf{Q}$ and the parabolicity of the evolution system with the operator $\mathbf{Q}$, see [9]. The latter two properties require a weaker condition $\lambda+2 \mu>0$.

Next assumptions concern the elastic energy:

(FE-1) Structure: $F(\boldsymbol{\epsilon}, \theta)$ is of class $C^{3}$ on $S^{2} \times[0, \infty)$, where $S^{2}$ denotes the set of symmetric tensors of second order in $\mathbb{R}^{n}$. We assume that

$$
F(\boldsymbol{\epsilon}, \theta)=F_{1}(\boldsymbol{\epsilon}, \theta)+F_{2}(\boldsymbol{\epsilon}),
$$

where $F_{1}(\boldsymbol{\epsilon}, \theta)$ is a concave function with respect to $\theta$,

$$
F_{1 / \theta \theta}(\boldsymbol{\epsilon}, \theta) \leq 0 \quad \text { for }(\boldsymbol{\epsilon}, \theta) \in S^{2} \times[0, \infty),
$$

such that $F_{1}(\boldsymbol{\epsilon}, \theta)$ is linear in $\theta$ over a certain interval $\left[0, \theta_{1}\right), \theta_{1}=$ const., and grows like $\theta^{r}$ for $\theta \geq \theta_{1}$.

(FE-2) Growth conditions: There exists a positive constant $\Lambda$ such that for $\theta \geq \theta_{1}$ and large values of $\epsilon_{i j}$ the following conditions are satisfied:

$$
\begin{aligned}
\left|F_{1 / \boldsymbol{\epsilon}}(\boldsymbol{\epsilon}, \theta)\right| & \leq \Lambda \theta^{r}|\boldsymbol{\epsilon}|^{q-1}, & \left|F_{2 / \boldsymbol{\epsilon}}(\boldsymbol{\epsilon})\right| & \leq \Lambda|\boldsymbol{\epsilon}|^{\bar{q}-1}, \\
\left|F_{1 / \boldsymbol{\epsilon} \theta}(\boldsymbol{\epsilon}, \theta)\right| & \leq \Lambda \theta^{r-1}|\boldsymbol{\epsilon}|^{q}, & \left|F_{1 / \theta \theta}(\boldsymbol{\epsilon}, \theta)\right| & \leq \Lambda \theta^{r-2}|\boldsymbol{\epsilon}|^{q+1}, \\
\left|F_{1 / \boldsymbol{\epsilon}}(\boldsymbol{\epsilon}, \theta)\right| & \leq \Lambda \theta^{r}|\boldsymbol{\epsilon}|^{q}, & \left|F_{2 / \boldsymbol{\epsilon}}(\boldsymbol{\epsilon})\right| & \leq \Lambda|\boldsymbol{\epsilon}|^{\bar{q}}
\end{aligned}
$$

with

$$
0<r<\frac{1}{2}, \quad 1<\bar{q} \leq \frac{q_{n} p_{n}}{4 n}, \quad 0<q \leq(\bar{q}+1)\left(\frac{1}{2}-r\right)
$$

where

$$
p_{n}=n+2
$$

and $q_{n}$ is the Sobolev exponent for which the imbedding of $W_{2}^{1}(\Omega)$ into $L_{q_{n}}(\Omega)$ is continuous, that is, $q_{n}=2 n /(n-2)$ for $n \geq 3$ and $q_{n}$ is any finite number for $n=2$. We note that

$$
0<q \leq \frac{q_{n} p_{n}}{2 n}\left(\frac{1}{2}-r\right)
$$

The above conditions imply the following growth of $F(\epsilon, \theta)$ :

$$
\left|F_{1}(\boldsymbol{\epsilon}, \theta)\right| \leq \Lambda+\Lambda \theta^{r}|\boldsymbol{\epsilon}|^{q+1}, \quad\left|F_{2}(\boldsymbol{\epsilon})\right| \leq \Lambda+\Lambda|\boldsymbol{\epsilon}|^{\bar{q}+1} .
$$

For example, in three-dimensional case the above conditions are satisfied for

$$
\bar{q}=\frac{5}{2}, \quad q=1, \quad r=\frac{3}{14} .
$$

Moreover, we assume the structural lower bound for the part $F_{2}(\boldsymbol{\epsilon})$ of the free energy.

(FE-3) There exist positive constants $c, \Lambda$ such that

$$
c|\boldsymbol{\epsilon}|^{\bar{q}+1}-\Lambda \leq F_{2}(\boldsymbol{\epsilon})
$$

The next assumption concerns the structural simplification of the energy equation by neglecting the nonlinear elastic contribution $-\theta F_{1 / \theta \theta}(\epsilon, \theta)$ in the specific heat coefficient. This allows to apply the classical parabolic theory in the existence proof. 
We point out that because of the applied technique we were unable either to allow $F_{1}(\epsilon, \theta)$ linear in $\theta$ or, assuming $\theta$-growth condition, to incorporate the arising nonlinearity in the specific heat coefficient.

(SH) The elastic energy contribution $-\theta F_{1 / \theta \theta}(\boldsymbol{\epsilon}, \theta)$ to the specific heat coefficient due to the nonlinearity of $F_{1}$ in $\theta$ is neglected, that is, we set

$$
c(\boldsymbol{\epsilon}, \theta)=c_{v}=\text { const. }>0 .
$$

We are looking for the solution in the Sobolev space

$$
V(p)=\left\{(\mathbf{u}, \theta) \in \mathbf{W}_{p}^{4,2}\left(Q_{T}\right) \times W_{p}^{2,1}\left(Q_{T}\right)\right\},
$$

with a parameter $p$ related to $L_{p}$-integrability. The assumptions on the initial data and the source terms correspond to this space.

(BV-p) The initial conditions satisfy for $1<p<\infty$ the inclusions

$$
\mathbf{u}_{0} \in \mathbf{W}_{p}^{4-2 / p}(\Omega), \quad \mathbf{u}_{1} \in \mathbf{W}_{p}^{2-2 / p}(\Omega), \quad 0 \leq \theta_{0} \in W_{p}^{2-2 / p}(\Omega),
$$

and the compatibility relations. The source terms satisfy

$$
\mathbf{b} \in \mathbf{L}_{p}\left(Q_{T}\right), \quad g \in L_{p}\left(Q_{T}\right), \quad g \geq 0 \quad \text { a.e. in } Q_{T} .
$$

The first main result concerns the existence of solutions to problem $(\mathrm{P})$.

TheOREM 2.1. Under assumptions (D), (LP), (FE-1)-(FE-3), (SH), (BV-p) and the condition $0<\sqrt{\kappa} \leq \nu$, there exists for $p_{n} \leq p<\infty$ a solution $(\mathbf{u}, \theta) \in V(p)$ to problem $(\mathrm{P})$ for any $T>0$. Moreover, $\theta \geq 0$ in $Q_{T}$, and the following a priori estimates hold:

$$
\|\mathbf{u}\|_{\mathbf{W}_{p}^{4,2}\left(Q_{T}\right)} \leq \Lambda, \quad\|\theta\|_{W_{p}^{2,1}\left(Q_{T}\right)} \leq \Lambda
$$

with a constant $\Lambda$ depending on the data of the problem, $\Omega$ and time $T$.

We note some properties of the solution which follow directly from the classical imbeddings.

COROLlaRY 2.1. For a solution to problem (P) the following holds: $\mathbf{u}, \nabla \mathbf{u}, \nabla^{2} \mathbf{u}$, $\mathbf{u}_{t}, \theta$ are Hölder continuous in $Q_{T}, \nabla^{3} \mathbf{u}, \nabla \mathbf{u}_{t}, \boldsymbol{\nabla} \theta \in \mathbf{L}_{p}\left(Q_{T}\right), p_{n} \leq p<\infty$, and

$$
\begin{gathered}
|\mathbf{u}|,|\boldsymbol{\nabla} \mathbf{u}|,\left|\nabla^{2} \mathbf{u}\right|,\left|\mathbf{u}_{t}\right| \leq \Lambda, \quad 0 \leq \theta \leq \Lambda \quad \text { in } Q_{T}, \\
\left\|\nabla^{3} \mathbf{u}\right\|_{\mathbf{L}_{p}\left(Q_{T}\right)},\left\|\nabla \mathbf{u}_{t}\right\|_{\mathbf{L}_{p}\left(Q_{T}\right)},\|\boldsymbol{\nabla} \theta\|_{\mathbf{L}_{p}\left(Q_{T}\right)} \leq \Lambda .
\end{gathered}
$$

In order to prove the uniqueness of the solution, the continuity property of $\nabla \mathbf{u}_{t}$ in $Q_{T}$ is needed. This holds provided $p>p_{n}$.

TheOREM 2.2. Let the assumptions of Theorem 2.1 be satisfied for

$$
p_{n}<p<\infty .
$$

Then the solution to the problem $(\mathrm{P})$ is unique for any $T>0$.

We collect now a priori bounds which follow from the imbeddings.

Corollary 2.2. The solution to problem $(\mathrm{P})$ has in case $p_{n}<p<\infty$ the following properties: $\nabla^{3} \mathbf{u}, \nabla \mathbf{u}_{t}, \nabla \theta$ are Hölder continuous in $Q_{T}$ and satisfy the bounds

$$
\left|\nabla^{3} \mathbf{u}\right|,\left|\nabla \mathbf{u}_{t}\right|,|\nabla \theta| \leq \Lambda \quad \text { in } Q_{T} .
$$


3. Existence proof. In the proof we shall use a parabolic decomposition of elasticity system (1.1) and the Leray-Schauder fixed point theorem.

The proof consists of several steps which are described below.

Step 1. Parabolic decomposition of (1.1). If we choose numbers $\alpha, \beta$ so that

$$
\alpha+\beta=\nu, \quad \alpha \beta=\frac{\kappa}{4},
$$

the system (1.1) with initial conditions (1.6) and boundary conditions (1.4) decomposes into the following systems of BVP's for a vector field w:

$$
\left\{\begin{aligned}
\mathbf{w}_{t}-\beta \mathbf{Q w} & =\boldsymbol{\nabla} \cdot F_{/ \boldsymbol{\epsilon}}(\boldsymbol{\epsilon}, \theta)+\mathbf{b} & & \text { in } Q_{T} \\
\mathbf{w}(0, \mathbf{x}) & =\mathbf{u}_{1}(\mathbf{x})-\alpha \mathbf{Q} \mathbf{u}_{0}(\mathbf{x}) & & \text { in } \Omega \\
\mathbf{w} & =0 & & \text { on } S_{T}
\end{aligned}\right.
$$

and the displacement $\mathbf{u}$ :

$$
\left\{\begin{aligned}
\mathbf{u}_{t}-\alpha \mathbf{Q u} & =\mathbf{w} & & \text { in } Q_{T} \\
\mathbf{u}(0, \mathbf{x}) & =\mathbf{u}_{0}(\mathbf{x}) & & \text { in } \Omega \\
\mathbf{u} & =0 & & \text { on } S_{T} .
\end{aligned}\right.
$$

The condition $0<\sqrt{\kappa} \leq \nu$ assures that $\alpha, \beta>0$.

System (3.1), (3.2) for $\mathbf{w}, \mathbf{u}$ is coupled to the BVP for $\theta$ :

$$
\left\{\begin{aligned}
c_{v} \theta_{t}-k \Delta \theta & =\theta F_{/ \theta \boldsymbol{\epsilon}}(\boldsymbol{\epsilon}, \theta): \boldsymbol{\epsilon}_{t}+\nu\left(\mathbf{A} \boldsymbol{\epsilon}_{t}\right): \boldsymbol{\epsilon}_{t}+g & & \text { in } Q_{T}, \\
\theta(0, \mathbf{x}) & =\theta_{0}(\mathbf{x}) & & \text { in } \Omega, \\
\boldsymbol{\nabla} \theta \cdot \mathbf{n} & =0 & & \text { on } S_{T} .
\end{aligned}\right.
$$

We note that a solution $(\mathbf{u}, \theta) \in V(p)$ to system (3.1)-(3.3) satisfies problem (P) under assumption ( $\mathrm{SH})$.

Step 2. To this system we apply the Leray-Schauder fixed point theorem, recalled here in one of the equivalent formulations for reader's convenience.

Theorem 3.1 (see [2]). Let $\mathcal{B}$ be a Banach space. Assume that $T:[0,1] \times \mathcal{B} \longrightarrow \mathcal{B}$ is a map with the following properties:

(i) For any fixed $\tau \in[0,1]$ the map $T(\tau, \cdot): \mathcal{B} \longrightarrow \mathcal{B}$ is completely continuous.

(ii) For every bounded subset $\mathcal{C}$ of $\mathcal{B}$, the family of maps $T(\cdot, \chi):[0,1] \longrightarrow \mathcal{B}, \chi \in \mathcal{C}$, is uniformly equicontinuous.

(iii) There is a bounded subset $\mathcal{C}$ of $\mathcal{B}$, such that any fixed point in $\mathcal{B}$ of $T(\tau, \cdot)$, $0 \leq \tau \leq 1$, is contained in $\mathcal{C}$.

(iv) $T(0, \cdot)$ has precisely one fixed point in $\mathcal{B}$.

Then $T(1, \cdot)$ has at least one fixed point in $\mathcal{B}$.

We define now the map $T_{\tau}$ from $V(p)$ into $V(p)$,

$$
T_{\tau}:(\overline{\mathbf{u}}, \bar{\theta}) \longrightarrow(\mathbf{u}, \theta), \quad \tau \in[0,1]
$$


by means of the following three problems:

$$
\begin{aligned}
& \left\{\begin{aligned}
\mathbf{w}_{t}-\beta \mathbf{Q w} & =\tau\left[\boldsymbol{\nabla} \cdot F_{/ \boldsymbol{\epsilon}}(\overline{\boldsymbol{\epsilon}}, \bar{\theta})+\mathbf{b}\right] & & \text { in } Q_{T}, \\
\mathbf{w}(0, \mathbf{x}) & =\tau\left[\mathbf{u}_{1}(\mathbf{x})-\alpha \mathbf{Q} \mathbf{u}_{0}(\mathbf{x})\right] & & \text { in } \Omega, \\
\mathbf{w} & =0 & & \text { on } S_{T},
\end{aligned}\right. \\
& \left\{\begin{aligned}
\mathbf{u}_{t}-\alpha \mathbf{Q u} & =\mathbf{w} & & \text { in } Q_{T}, \\
\mathbf{u}(0, \mathbf{x}) & =\tau \mathbf{u}_{0}(\mathbf{x}) & & \text { in } \Omega \\
\mathbf{u} & =0 & & \text { on } S_{T},
\end{aligned}\right. \\
& \left\{\begin{aligned}
c_{v} \theta_{t}-k \Delta \theta & =\tau\left[\bar{\theta} F_{/ \theta \boldsymbol{\epsilon}}(\boldsymbol{\epsilon}, \bar{\theta}): \boldsymbol{\epsilon}_{t}+\nu\left(\mathbf{A} \boldsymbol{\epsilon}_{t}\right): \boldsymbol{\epsilon}_{t}+g\right] & & \text { in } Q_{T}, \\
\theta(0, \mathbf{x}) & =\tau \theta_{0}(\mathbf{x}) & & \text { in } \Omega, \\
\boldsymbol{\nabla} \theta \cdot \mathbf{n} & =0 & & \text { on } S_{T},
\end{aligned}\right.
\end{aligned}
$$

where $\overline{\boldsymbol{\epsilon}}=\boldsymbol{\epsilon}(\overline{\mathbf{u}})$.

Clearly, a fixed point of $T_{1}$ in $V(p)$ is equivalent to a solution $(\mathbf{u}, \theta)$ in $V(p)$ of the system (3.1)-(3.3), and thus is a solution to problem $(\mathrm{P})$ in $V(p)$.

In further steps of the proof we shall verify the assumptions of Theorem 3.1.

Step 3. It may be shown, see [9], that $T_{\tau}$ is well defined, i.e. $T_{\tau}(V(p)) \subset V(p)$, and is equicontinuous with respect to $\tau$. In addition, a null solution is the unique fixed point for $\tau=0$.

Step 4. It remains to verify assumption (iii) in the Leray-Schauder theorem, that is, to find an a priori bound for a fixed point of $T_{\tau}$. Without loss of generality we may set $\tau=1$. Let then $(\mathbf{u}, \theta) \in V(p), p_{n} \leq p<\infty$, be a fixed point of $T_{1}$.

First we recall the result of $[9]$ :

Lemma 3.1. If $(\mathbf{u}, \theta)$ is a fixed point of $T_{1}$ in $V(p), p_{n} \leq p<\infty$, then $\theta \geq 0$ in $Q_{T}$.

The proof that $(\mathbf{u}, \theta)$ is a priori bounded in $V(p)$ requires a sequence of estimates which will be iteratively improved. The first are, as usual, the energy estimates.

Lemma 3.2. A fixed point of $T_{1}$ satisfies for any $t \in I$ the following bound

$$
\int_{\Omega_{t}}\left(c_{v} \theta+\frac{\gamma}{2}\left|\mathbf{u}_{t}\right|^{2}+\frac{\gamma \kappa}{8}|\mathbf{Q u}|^{2}+\gamma c|\boldsymbol{\epsilon}|^{\bar{q}+1}\right) d x+\frac{1}{2} a_{\star} \nu(\gamma-1) \int_{Q_{t}}\left|\boldsymbol{\epsilon}_{t}\right|^{2} d x d t^{\prime} \leq \Lambda
$$

with some positive constants $c, \gamma>1$, and $\Lambda$ depending on the initial data, the sources b, $g$ and time horizon $T$.

PROOF. Integrating temperature equation in (3.3) over $Q_{t}$ and using boundary conditions gives

$$
\begin{aligned}
c_{v} \int_{Q_{t}} \frac{d}{d t} \theta & d x d t^{\prime} \\
& =\int_{Q_{t}} \theta F_{1 / \theta \boldsymbol{\epsilon}}(\boldsymbol{\epsilon}, \theta): \boldsymbol{\epsilon}_{t} d x d t^{\prime}+\nu \int_{Q_{t}}\left(\mathbf{A} \boldsymbol{\epsilon}_{t}\right): \boldsymbol{\epsilon}_{t} d x d t^{\prime}+\int_{Q_{t}} g d x d t^{\prime} .
\end{aligned}
$$


Multiplying the elasticity equation (1.1) by $\gamma \mathbf{u}_{t}, \gamma=$ const. $>1$ and integrating over $Q_{t}$ gives

$$
\begin{aligned}
\frac{\gamma}{2} \int_{Q_{t}} \frac{d}{d t}\left|\mathbf{u}_{t}\right|^{2} d x d t^{\prime}- & \gamma \nu \int_{Q_{t}}\left(\mathbf{Q u} \mathbf{u}_{t}\right) \cdot \mathbf{u}_{t} d x d t^{\prime}+\frac{\gamma \kappa}{4} \int_{Q_{t}}(\mathbf{Q Q u}) \cdot \mathbf{u}_{t} d x d t^{\prime} \\
& -\gamma \int_{Q_{t}}\left(\boldsymbol{\nabla} \cdot F_{/ \epsilon}(\boldsymbol{\epsilon}, \theta)\right) \cdot \mathbf{u}_{t} d x d t^{\prime}=\gamma \int_{Q_{t}} \mathbf{b} \cdot \mathbf{u}_{t} d x d t^{\prime}
\end{aligned}
$$

The second integral on the left-hand side of (3.6), after integration by parts and using the boundary condition for $\mathbf{u}$, is

$$
-\gamma \nu \int_{Q_{t}}\left(\mathbf{Q u} \mathbf{u}_{t}\right) \cdot \mathbf{u}_{t} d x d t^{\prime}=\gamma \nu \int_{Q_{t}}\left(\mathbf{A} \boldsymbol{\epsilon}_{t}\right): \boldsymbol{\epsilon}_{t} d x d t^{\prime} .
$$

The third integral, after applying the integration by parts and the boundary condition for $\mathbf{Q u}$, becomes

$$
\text { (3.8) } \frac{\gamma \kappa}{4} \int_{Q_{t}}(\mathbf{Q Q u}) \cdot \mathbf{u}_{t} d x d t^{\prime}=\frac{\gamma \kappa}{4} \int_{Q_{t}}(\mathbf{Q u}) \cdot\left(\mathbf{Q u} \mathbf{u}_{t}\right) d x d t^{\prime}=\frac{\gamma \kappa}{8} \int_{Q_{t}} \frac{d}{d t}|\mathbf{Q u}|^{2} d x d t^{\prime} .
$$

Finally, by integrating by parts and using the boundary condition for $\mathbf{u}$, the fourth integral gives

$$
\begin{aligned}
&-\gamma \int_{Q_{t}}\left(\nabla \cdot F_{/ \epsilon}(\boldsymbol{\epsilon}, \theta)\right) \cdot \mathbf{u}_{t} d x d t^{\prime}=\gamma \int_{Q_{t}} F_{/ \epsilon}(\boldsymbol{\epsilon}, \theta): \boldsymbol{\epsilon}_{t} d x d t^{\prime} \\
&=\gamma \int_{Q_{t}} \frac{d}{d t} F_{2}(\boldsymbol{\epsilon}) d x d t^{\prime}+\gamma \int_{Q_{t}} F_{1 / \epsilon}(\boldsymbol{\epsilon}, \theta): \boldsymbol{\epsilon}_{t} d x d t^{\prime} .
\end{aligned}
$$

Using (3.7)-(3.9) in (3.6) and combining with (3.5) gives the identity

$$
\begin{aligned}
\int_{Q_{t}} \frac{d}{d t}\left(c_{v} \theta+\right. & \left.\frac{\gamma}{2}\left|\mathbf{u}_{t}\right|^{2}+\frac{\gamma \kappa}{8}|\mathbf{Q u}|^{2}+\gamma F_{2}(\boldsymbol{\epsilon})\right) d x d t^{\prime}+\nu(\gamma-1) \int_{Q_{t}}\left(\mathbf{A} \epsilon_{t}\right): \boldsymbol{\epsilon}_{t} d x d t^{\prime} \\
= & \int_{Q_{t}}\left(\theta F_{1 / \theta \boldsymbol{\epsilon}}(\boldsymbol{\epsilon}, \theta)-\gamma F_{1 / \epsilon}(\boldsymbol{\epsilon}, \theta)\right): \boldsymbol{\epsilon}_{t} d x d t^{\prime}+\int_{Q_{t}}\left(g+\gamma \mathbf{b} \cdot \mathbf{u}_{t}\right) d x d t^{\prime}
\end{aligned}
$$

Hence, using assumption (FE-3) and the bound (1.8) we obtain

$$
\begin{array}{r}
\int_{\Omega_{t}}\left(c_{v} \theta+\frac{\gamma}{2}\left|\mathbf{u}_{t}\right|^{2}+\frac{\gamma \kappa}{8}|\mathbf{Q u}|^{2}+\gamma c|\boldsymbol{\epsilon}|^{\bar{q}+1}\right) d x+a_{\star} \nu(\gamma-1) \int_{Q_{t}}\left|\boldsymbol{\epsilon}_{t}\right|^{2} d x d t^{\prime} \\
\leq \Lambda+\int_{\Omega}\left(c_{v} \theta_{0}+\frac{\gamma}{2}\left|\mathbf{u}_{1}\right|^{2}+\frac{\gamma \kappa}{8}\left|\mathbf{Q} \mathbf{u}_{0}\right|^{2}+\gamma F_{2}\left(\boldsymbol{\epsilon}_{0}\right)\right) d x \\
+\int_{Q_{t}}\left(g+\frac{\gamma}{2}|\mathbf{b}|^{2}\right) d x d t^{\prime}+\int_{Q_{t}} \frac{\gamma}{2}\left|\mathbf{u}_{t}\right|^{2} d x d t^{\prime} \\
+\int_{Q_{t}}\left(\theta F_{1 / \theta \boldsymbol{\epsilon}}(\boldsymbol{\epsilon}, \theta)-\gamma F_{1 / \boldsymbol{\epsilon}}(\boldsymbol{\epsilon}, \theta)\right): \boldsymbol{\epsilon}_{t} d x d t^{\prime},
\end{array}
$$

where $\epsilon_{0}=\epsilon\left(\mathbf{u}_{0}\right)$.

By Young's inequality the last integral on the right-hand side of (3.10) is estimated 
by

$$
\begin{aligned}
\int_{Q_{t}}\left(\theta F_{1 / \theta \boldsymbol{\epsilon}}(\boldsymbol{\epsilon}, \theta)\right. & \left.-\gamma F_{1 / \boldsymbol{\epsilon}}(\boldsymbol{\epsilon}, \theta)\right): \boldsymbol{\epsilon}_{t} d x d t^{\prime} \\
\leq & \frac{\delta}{2} \int_{Q_{t}}\left|\boldsymbol{\epsilon}_{t}\right|^{2} d x d t^{\prime}+\frac{1}{2 \delta} \int_{Q_{t}}\left|\theta F_{1 / \theta \boldsymbol{\epsilon}}(\boldsymbol{\epsilon}, \theta)-\gamma F_{1 / \boldsymbol{\epsilon}}(\boldsymbol{\epsilon}, \theta)\right|^{2} d x d t^{\prime}
\end{aligned}
$$

where, with the appropriate choice of $\delta, \delta$-integral is absorbed by left-hand side of (3.10).

Applying assumptions (FE-1), (FE-2) to the second integral on the right-hand side of (3.11) gives

$$
\begin{gathered}
\int_{Q_{t}}\left|\theta F_{1 / \theta \boldsymbol{\epsilon}}(\boldsymbol{\epsilon}, \theta)-\gamma F_{1 / \boldsymbol{\epsilon}}(\boldsymbol{\epsilon}, \theta)\right|^{2} d x d t^{\prime} \leq \Lambda+\Lambda \int_{Q_{t}} \theta^{2 r}|\boldsymbol{\epsilon}|^{2 q} d x d t^{\prime} \\
\leq \Lambda+\frac{\Lambda}{p_{1}} \int_{Q_{t}} \theta^{2 r p_{1}} d x d t^{\prime}+\frac{\Lambda}{p_{2}} \int_{Q_{t}}|\boldsymbol{\epsilon}|^{2 q p_{2}} d x d t^{\prime} \\
\leq \Lambda+\frac{\Lambda}{p_{1}} \int_{Q_{t}} \theta d x d t^{\prime}+\frac{\Lambda}{p_{2}} \int_{Q_{t}}|\boldsymbol{\epsilon}|^{2 q /(1-2 r)} d x d t^{\prime}
\end{gathered}
$$

where we have used Young's inequality with $p_{1}=1 /(2 r), p_{2}=1 /(1-2 r)$.

By the condition on $q$ the last integral in (3.12) is estimated by

$$
\int_{Q_{t}}|\boldsymbol{\epsilon}|^{2 q /(1-2 r)} d x d t^{\prime} \leq \int_{Q_{t}}|\boldsymbol{\epsilon}|^{\bar{q}+1} d x d t^{\prime} .
$$

Consequently, combining (3.10) and (3.11)-(3.13) gives

$$
\begin{array}{r}
\int_{\Omega_{t}}\left(c_{v} \theta+\frac{\gamma}{2}\left|\mathbf{u}_{t}\right|^{2}+\frac{\gamma \kappa}{8}|\mathbf{Q u}|^{2}+\gamma c|\boldsymbol{\epsilon}|^{\bar{q}+1}\right) d x+\frac{1}{2} a_{\star} \nu(\gamma-1) \int_{Q_{t}}\left|\boldsymbol{\epsilon}_{t}\right|^{2} d x d t^{\prime} \\
\leq \Lambda+\Lambda \int_{Q_{t}}\left(\theta+\left|\mathbf{u}_{t}\right|^{2}+|\boldsymbol{\epsilon}|^{\bar{q}+1}\right) d x d t^{\prime}
\end{array}
$$

Since, by Lemma 3.1, $\theta \geq 0$, applying Gronwall's inequality in (3.14) yields the assertion.

The energy estimates allow us to obtain more refined bounds for the fixed point. By the strong ellipticity property of the operator $\mathbf{Q}$ and the result of Nečas [7] it follows from (3.4) that

$$
\|\mathbf{u}\|_{L_{\infty}\left(I ; \mathbf{W}_{2}^{2}(\Omega)\right)} \leq \Lambda
$$

Consequently,

$$
\|\boldsymbol{\epsilon}\|_{L_{\infty}\left(I ; \mathbf{W}_{2}^{1}(\Omega)\right)} \leq \Lambda
$$

and by the imbedding,

$$
\|\boldsymbol{\epsilon}\|_{L_{\infty}\left(I ; \mathbf{L}_{q_{n}}(\Omega)\right)} \leq \Lambda
$$

Moreover, (3.4) gives

$$
\mathbf{u} \in \mathbf{W}_{2, \infty}^{2,1}\left(Q_{T}\right) \quad \text { and } \quad\|\mathbf{u}\|_{\mathbf{W}_{2, \infty}^{2,1}\left(Q_{T}\right)} \leq \Lambda .
$$

Hence,

$$
\boldsymbol{\epsilon} \in \mathbf{W}_{2, \infty}^{1,1 / 2}\left(Q_{T}\right) \quad \text { and } \quad\|\boldsymbol{\epsilon}\|_{\mathbf{W}_{2, \infty}^{1,1 / 2}\left(Q_{T}\right)} \leq \Lambda
$$


so, by the imbedding,

$$
\boldsymbol{\epsilon} \in \mathbf{L}_{p}\left(Q_{T}\right) \quad \text { and } \quad\|\boldsymbol{\epsilon}\|_{\mathbf{L}_{p}\left(Q_{T}\right)} \leq \Lambda \quad \text { for } \quad p=\frac{2 p_{n}}{n-2}=\frac{q_{n} p_{n}}{n} .
$$

Our strategy now is to improve the estimates for $\epsilon$. To this purpose we use the representation of solutions to parabolic systems with the right-hand side in the divergence form (see Lemma 7.2 in [9]). Applied to solutions of BVP (3.1) it gives

$$
\mathbf{w}-\mathbf{w}(0)=\mathbf{w}^{0}+\sum_{i=1}^{n} \frac{\partial \mathbf{w}^{i}}{\partial x_{i}}
$$

where $\mathbf{w}(0)=\mathbf{u}_{1}-\alpha \mathbf{Q} \mathbf{u}_{0}$, and $\mathbf{w}^{i}, i=0,1, \ldots, n$ are the solutions of the problems

$$
\begin{aligned}
& \mathbf{w}_{t}^{i}-\beta \mathbf{Q} \mathbf{w}^{i}=\mathbf{h}^{i} \quad \text { in } Q_{T}, \\
& \mathbf{w}^{i}(0, \mathbf{x})=0 \quad \text { in } \Omega, \quad 0 \leq i \leq n, \\
& \mathbf{w}^{i}=0 \quad \text { on } S_{T} \quad \text { for } 0 \leq i \leq n-1, \\
& \frac{\partial \mathbf{w}^{n}}{\partial \mathbf{n}}=0 \quad \text { on } S_{T} \quad \text { for } i=n,
\end{aligned}
$$

with $\mathbf{h}^{0}=\mathbf{b}+\beta \mathbf{Q w}(0), \mathbf{h}^{i}=\left(F_{/ \epsilon_{k i}}(\boldsymbol{\epsilon}, \theta)\right)_{k=1, \ldots, n}$.

According to the lemma mentioned above they satisfy the estimate

$$
\|\mathbf{w}-\mathbf{w}(0)\|_{\mathbf{W}_{p}^{1,1 / 2}\left(Q_{t}\right)} \leq \Lambda\left(\|\mathbf{b}\|_{\mathbf{L}_{p}\left(Q_{t}\right)}+\|\beta \mathbf{Q W}(0)\|_{\mathbf{L}_{p}(\Omega)}+\left\|F_{/ \boldsymbol{\epsilon}}(\boldsymbol{\epsilon}, \theta)\right\|_{\mathbf{L}_{p}\left(Q_{t}\right)}\right)
$$

with the constant $\Lambda$ depending on $p, T, \Omega$.

We start by utilizing (3.16) for $p=4$. To this purpose we estimate $\left\|F_{/ \boldsymbol{\epsilon}}(\boldsymbol{\epsilon}, \theta)\right\|_{\mathbf{L}_{4}\left(Q_{t}\right)}$ in terms of the norm $\|\theta\|_{L_{2}\left(Q_{t}\right)}$ which will be bounded later on in Lemma 3.4.

LEMMA 3.3. The following inequality holds:

$$
\left\|F_{/ \epsilon}(\boldsymbol{\epsilon}, \theta)\right\|_{\mathbf{L}_{4}\left(Q_{t}\right)} \leq \Lambda+\Lambda\|\theta\|_{L_{2}\left(Q_{t}\right)}^{1 / 2} .
$$

ProOF. We have

$$
\left\|F_{/ \boldsymbol{\epsilon}}(\boldsymbol{\epsilon}, \theta)\right\|_{\mathbf{L}_{4}\left(Q_{t}\right)}^{4} \leq \Lambda\left(\left\|F_{1 / \boldsymbol{\epsilon}}(\boldsymbol{\epsilon}, \theta)\right\|_{\mathbf{L}_{4}\left(Q_{t}\right)}^{4}+\left\|F_{2 / \boldsymbol{\epsilon}}(\boldsymbol{\epsilon})\right\|_{\mathbf{L}_{4}\left(Q_{t}\right)}^{4}\right) .
$$

Applying the growth condition (FE-2) and estimate (3.15) gives

$$
\begin{aligned}
\left\|F_{1 / \boldsymbol{\epsilon}}(\boldsymbol{\epsilon}, \theta)\right\|_{\mathbf{L}_{4}\left(Q_{t}\right)}^{4} & \leq \Lambda+\Lambda \int_{Q_{t}} \theta^{4 r}|\boldsymbol{\epsilon}|^{4 q} d x d t^{\prime} \\
& \leq \Lambda+\frac{\Lambda}{p_{1}} \int_{Q_{t}} \theta^{4 r p_{1}} d x d t^{\prime}+\frac{\Lambda}{p_{2}} \int_{Q_{t}}|\boldsymbol{\epsilon}|^{4 q p_{2}} d x d t^{\prime} \\
& \leq \Lambda+\frac{\Lambda}{p_{1}} \int_{Q_{t}} \theta^{2} d x d t^{\prime}+\frac{\Lambda}{p_{2}} \int_{Q_{t}}|\boldsymbol{\epsilon}|^{4 q /(1-2 r)} d x d t^{\prime}
\end{aligned}
$$

for $p_{1}=1 /(2 r), p_{2}=1 /(1-2 r)<\infty$. Since, by the assumption on $q$,

$$
\frac{4 q}{1-2 r} \leq \frac{q_{n} p_{n}}{n}
$$

the last integral in (3.19) is, due to (3.15), bounded by a constant $\Lambda$. Similarly, recalling the assumption on $\bar{q}$, we get

$$
\left\|F_{2 / \boldsymbol{\epsilon}}(\boldsymbol{\epsilon})\right\|_{\mathbf{L}_{4}\left(Q_{t}\right)}^{4} \leq \Lambda+\Lambda \int_{Q_{t}}|\boldsymbol{\epsilon}|^{4 \bar{q}} d x d t^{\prime} \leq \Lambda+\Lambda \int_{Q_{t}}|\boldsymbol{\epsilon}|^{q_{n} p_{n} / n} d x d t^{\prime} \leq \Lambda .
$$


Combining (3.18), (3.19) and (3.21) gives estimate (3.17).

By virtue of (3.17) and by assumption (BV-p) it follows from (3.16) that

$$
\|\mathbf{w}\|_{\mathbf{W}_{4}^{1,1 / 2}\left(Q_{t}\right)} \leq \Lambda+\Lambda\|\theta\|_{L_{2}\left(Q_{t}\right)}^{1 / 2} .
$$

With this, using the regularity property of parabolic systems (see Lemma 7.3 [9]) we get

$$
\|\nabla \mathbf{u}\|_{\mathbf{W}_{4}^{2,1}\left(Q_{t}\right)} \leq \Lambda+\Lambda\|\theta\|_{L_{2}\left(Q_{t}\right)}^{1 / 2},
$$

so

$$
\|\boldsymbol{\epsilon}\|_{\mathbf{W}_{4}^{2,1}\left(Q_{t}\right)} \leq \Lambda+\Lambda\|\theta\|_{L_{2}\left(Q_{t}\right)}^{1 / 2} .
$$

With this estimate we are ready to prove the temperature bounds.

LEMMA 3.4. If (3.22) holds then there exists a constant $\Lambda$ depending on the data of the problem such that the solution of BVP (3.3) satisfies for any $t \in I$ the estimate

$$
\int_{\Omega_{t}} \theta^{2} d x+\int_{Q_{t}}|\nabla \theta|^{2} d x d t^{\prime} \leq \Lambda
$$

Proof. We multiply the temperature equation (3.3) by $\theta$ and integrate over $Q_{t}$ using the boundary conditions to get

$$
\begin{aligned}
\frac{c_{v}}{2} \int_{Q_{t}} \frac{d}{d t} \theta^{2} d x d t^{\prime}+k \int_{Q_{t}}|\nabla \theta|^{2} d x d t^{\prime} & =\int_{Q_{t}} \theta^{2} F_{1 / \theta \boldsymbol{\epsilon}}(\boldsymbol{\epsilon}, \theta): \boldsymbol{\epsilon}_{t} d x d t^{\prime} \\
& +\nu \int_{Q_{t}} \theta\left(\mathbf{A} \boldsymbol{\epsilon}_{t}\right): \boldsymbol{\epsilon}_{t} d x d t^{\prime}+\int_{Q_{t}} \theta g d x d t^{\prime} .
\end{aligned}
$$

Using Young's inequality the first integral on the right-hand side of (3.24) is estimated by

$$
\int_{Q_{t}} \theta^{2} F_{1 / \theta \boldsymbol{\epsilon}}(\boldsymbol{\epsilon}, \theta): \boldsymbol{\epsilon}_{t} d x d t^{\prime} \leq \frac{1}{4} \int_{Q_{t}}\left|\boldsymbol{\epsilon}_{t}\right|^{4} d x d t^{\prime}+\frac{3}{4} \int_{Q_{t}}\left|\theta^{2} F_{1 / \theta \boldsymbol{\epsilon}}(\boldsymbol{\epsilon}, \theta)\right|^{4 / 3} d x d t^{\prime},
$$

where, by (3.22), the $\epsilon_{t}$-integral is bounded by

$$
\Lambda+\Lambda \int_{Q_{t}} \theta^{2} d x d t^{\prime}
$$

and for the second integral, using the growth condition, we have

$$
\begin{aligned}
& \int_{Q_{t}}\left|\theta^{2} F_{1 / \theta \boldsymbol{\epsilon}}(\boldsymbol{\epsilon}, \theta)\right|^{4 / 3} d x d t^{\prime} \leq \Lambda+\int_{Q_{t}} \theta^{4(r+1) / 3}|\boldsymbol{\epsilon}|^{4 q / 3} d x d t^{\prime} \\
& \leq \Lambda+\frac{\Lambda}{p_{1}} \int_{Q_{t}} \theta^{4(r+1) p_{1} / 3} d x d t^{\prime}+\frac{\Lambda}{p_{2}} \int_{Q_{t}}|\boldsymbol{\epsilon}|^{4 q p_{2} / 3} d x d t^{\prime} \\
& \leq \Lambda+\frac{\Lambda}{p_{1}} \int_{Q_{t}} \theta^{2} d x d t^{\prime}+\frac{\Lambda}{p_{2}} \int_{Q_{t}}|\boldsymbol{\epsilon}|^{4 q /(1-2 r)} d x d t^{\prime}
\end{aligned}
$$

for $p_{1}=3 /(2 r+2), p_{2}=3 /(1-2 r)$.

By recalling (3.20) and estimate (3.15), the right-hand side of (3.26) is bounded by the expression (3.25). 
Now, using (3.22), we estimate the second integral on the right-hand side of (3.24) as follows

$$
\int_{Q_{t}} \theta\left(\mathbf{A} \boldsymbol{\epsilon}_{t}\right): \boldsymbol{\epsilon}_{t} d x d t^{\prime} \leq \Lambda \int_{Q_{t}}\left|\boldsymbol{\epsilon}_{t}\right|^{4} d x d t^{\prime}+\Lambda \int_{Q_{t}} \theta^{2} d x d t^{\prime} \leq \Lambda+\Lambda \int_{Q_{t}} \theta^{2} d x d t^{\prime}
$$

Clearly, the last term in (3.24) is also majorized by (3.25). Returning to (3.24) and incorporating the above gives

$$
\frac{c_{v}}{2} \int_{\Omega_{t}} \theta^{2} d x+k \int_{Q_{t}}|\nabla \theta|^{2} d x d t^{\prime} \leq \frac{c_{v}}{2} \int_{\Omega} \theta_{0}^{2} d x+\Lambda+\Lambda \int_{Q_{t}} \theta^{2} d x d t^{\prime} .
$$

Now the application of Gronwall's inequality yields the assertion.

Utilizing temperature estimates (3.23) in (3.22) gives the bound

$$
\|\boldsymbol{\epsilon}\|_{\mathbf{W}_{4}^{2,1}\left(Q_{t}\right)} \leq \Lambda \text {. }
$$

Hence, by the imbedding, $\epsilon$ is Hölder continuous in $Q_{T}$, and

$$
|\boldsymbol{\epsilon}| \leq \Lambda \quad \text { in } Q_{T} .
$$

Moreover, by the imbedding of the space $L_{\infty}\left(I ; L_{2}(\Omega)\right) \cap L_{2}\left(I ; W_{2}^{1}(\Omega)\right)$ in $L_{p}\left(Q_{t}\right)$ for $p>2$ it follows from $(3.23)$ that

$$
\|\theta\|_{L_{2 p_{n} / n}\left(Q_{t}\right)} \leq \Lambda \text {. }
$$

Thanks to (3.27) and (3.28), in 3-D case we can further improve the estimates. Now we have

$$
\left\|F_{/ \boldsymbol{\epsilon}}(\boldsymbol{\epsilon}, \theta)\right\|_{\mathbf{L}_{p_{n}}\left(Q_{t}\right)} \leq \Lambda
$$

which results from the estimates

$$
\begin{aligned}
\int_{Q_{t}}\left|F_{1 / \epsilon}(\boldsymbol{\epsilon}, \theta)\right|^{p_{n}} d x d t^{\prime} & \leq \Lambda+\frac{\Lambda}{p_{1}} \int_{Q_{t}} \theta^{r p_{n} p_{1}} d x d t^{\prime}+\frac{\Lambda}{p_{2}} \int_{Q_{t}}|\boldsymbol{\epsilon}|^{q p_{n} p_{2}} d x d t^{\prime} \\
& \leq \Lambda+\frac{\Lambda}{p_{1}} \int_{Q_{t}} \theta^{2 p_{n} / n} d x d t^{\prime}+\frac{\Lambda}{p_{2}} \int_{Q_{t}}|\boldsymbol{\epsilon}|^{2 q p_{n} /(2-n r)} d x d t^{\prime} \leq \Lambda
\end{aligned}
$$

for $p_{1}=2 /(n r), p_{2}=2 /(2-n r)$, and

$$
\int_{Q_{t}}\left|F_{2 / \boldsymbol{\epsilon}}(\boldsymbol{\epsilon})\right|^{p_{n}} d x d t^{\prime} \leq \Lambda+\Lambda \int_{Q_{t}}|\boldsymbol{\epsilon}|^{\bar{q} p_{n}} d x d t^{\prime} \leq \Lambda .
$$

So, returning to $(3.16)$ gives

and subsequently,

$$
\|\mathbf{w}\|_{\mathbf{W}_{p_{n}}^{1,1 / 2}\left(Q_{t}\right)} \leq \Lambda
$$

$$
\|\boldsymbol{\epsilon}\|_{\mathbf{W}_{p_{n}}^{2,1}\left(Q_{T}\right)} \leq \Lambda \text {. }
$$

By the imbedding, we conclude from (3.30) that

$$
\nabla \boldsymbol{\epsilon} \in \mathbf{L}_{p}\left(Q_{t}\right) \quad \text { and } \quad\|\nabla \boldsymbol{\epsilon}\|_{\mathbf{L}_{p}\left(Q_{t}\right)} \leq \Lambda \text { for } p_{n} \leq p<\infty .
$$

Our further procedure consists in applying to BVP (3.3) the classical parabolic theory ([6], Thm. IV.9.1). We have 
LEмma 3.5. The following bound holds for the right-hand side of the temperature equation (3.3):

$$
\left\|\theta F_{1 / \theta \boldsymbol{\epsilon}}(\boldsymbol{\epsilon}, \theta): \boldsymbol{\epsilon}_{t}+\nu\left(\mathbf{A} \boldsymbol{\epsilon}_{t}\right): \boldsymbol{\epsilon}_{t}+g\right\|_{L_{p_{n} / 2}\left(Q_{t}\right)} \leq \Lambda
$$

Proof. We have

$$
\int_{Q_{t}}\left|\theta F_{1 / \theta \boldsymbol{\epsilon}}(\boldsymbol{\epsilon}, \theta): \boldsymbol{\epsilon}_{t}\right|^{p_{n} / 2} d x d t^{\prime} \leq \frac{1}{2} \int_{Q_{t}}\left|\boldsymbol{\epsilon}_{t}\right|^{p_{n}} d x d t^{\prime}+\frac{1}{2} \int_{Q_{t}}\left|\theta F_{1 / \theta \boldsymbol{\epsilon}}(\boldsymbol{\epsilon}, \theta)\right|^{p_{n}} d x d t^{\prime},
$$

where the first term on the right-hand side is, by (3.30), bounded by $\Lambda$. For the second one we have

$$
\begin{aligned}
\int_{Q_{t}}\left|\theta F_{1 / \theta \boldsymbol{\epsilon}}(\boldsymbol{\epsilon}, \theta)\right|^{p_{n}} d x d t^{\prime} & \leq \Lambda+\Lambda \int_{Q_{t}} \theta^{r p_{n}}|\boldsymbol{\epsilon}|^{q p_{n}} d x d t^{\prime} \\
& \leq \Lambda+\frac{\Lambda}{p_{1}} \int_{Q_{t}} \theta^{r p_{n} p_{1}} d x d t^{\prime}+\frac{\Lambda}{p_{2}} \int_{Q_{t}}|\boldsymbol{\epsilon}|^{q p_{n} p_{2}} d x d t^{\prime} \\
& \leq \Lambda+\frac{\Lambda}{p_{1}} \int_{Q_{t}} \theta^{2 p_{n} / n} d x d t^{\prime}+\frac{\Lambda}{p_{2}} \int_{Q_{t}}|\boldsymbol{\epsilon}|^{2 q p_{n} /(2-n r)} d x d t^{\prime} \leq \Lambda
\end{aligned}
$$

for $p_{1}=2 /(n r), p_{2}=2 /(2-n r)$, where we have used the bounds (3.28) and (3.27).

Similarly, utilizing (3.30), we have

$$
\int_{Q_{t}}\left|\left(\mathbf{A} \boldsymbol{\epsilon}_{t}\right): \boldsymbol{\epsilon}_{t}\right|^{p_{n} / 2} d x d t^{\prime} \leq \frac{1}{2} \int_{Q_{t}}\left|\mathbf{A} \boldsymbol{\epsilon}_{t}\right|^{p_{n}} d x d t^{\prime}+\frac{1}{2} \int_{Q_{t}}\left|\boldsymbol{\epsilon}_{t}\right|^{p_{n}} d x d t^{\prime} \leq \Lambda .
$$

This shows the assertion.

The bound (3.32) together with the parabolic theory imply that

$$
\theta \in W_{p_{n} / 2}^{2,1}\left(Q_{t}\right) \quad \text { and } \quad\|\theta\|_{W_{p_{n} / 2}^{2,1}\left(Q_{t}\right)} \leq \Lambda
$$

so, by the imbedding,

$$
\nabla \theta \in \mathbf{L}_{p_{n}}\left(Q_{t}\right), \quad \theta \in L_{p}\left(Q_{t}\right)
$$

and

$$
\|\nabla \theta\|_{\mathbf{L}_{p_{n}}\left(Q_{t}\right)} \leq \Lambda, \quad\|\theta\|_{L_{p}\left(Q_{t}\right)} \leq \Lambda \quad \text { for } \quad \frac{p_{n}}{2} \leq p<\infty .
$$

Now we are ready to improve iteratively a priori bounds. To this purpose we return to the decomposed system and estimate the right-hand side of the w-equation (3.1).

LEMma 3.6. The following bound holds for the right-hand side of (3.1):

$$
\left\|\nabla \cdot F_{/ \epsilon}(\boldsymbol{\epsilon}, \theta)\right\|_{\mathbf{L}_{p_{n}}\left(Q_{t}\right)} \leq \Lambda
$$

Proof. Applying the bound

$$
\left\|\nabla \cdot F_{/ \boldsymbol{\epsilon}}(\boldsymbol{\epsilon}, \theta)\right\|_{\mathbf{L}_{p_{n}}\left(Q_{t}\right)} \leq\left\|\boldsymbol{\nabla} \cdot F_{1 / \boldsymbol{\epsilon}}(\boldsymbol{\epsilon}, \theta)\right\|_{\mathbf{L}_{p_{n}}\left(Q_{t}\right)}+\left\|\nabla \cdot F_{2 / \boldsymbol{\epsilon}}(\boldsymbol{\epsilon})\right\|_{\mathbf{L}_{p_{n}}\left(Q_{t}\right)},
$$

we get

$$
\begin{aligned}
\int_{Q_{t}}\left|\nabla \cdot F_{1 / \epsilon}(\boldsymbol{\epsilon}, \theta)\right|^{p_{n}} d x d t^{\prime} \leq \Lambda \int_{Q_{t}}\left|F_{1 / \epsilon_{i j} \epsilon_{k l}}(\boldsymbol{\epsilon}, \theta) \partial_{j} \epsilon_{k l}\right|^{p_{n}} d x d t^{\prime} \\
\quad+\Lambda \int_{Q_{t}}\left|F_{1 / \epsilon_{i j} \theta}(\epsilon, \theta) \partial_{j} \theta\right|^{p_{n}} d x d t^{\prime} \equiv I_{1}+I_{2} .
\end{aligned}
$$


By recalling the growth conditions, the term $I_{1}$ is estimated by

$$
\begin{aligned}
I_{1} \leq \Lambda \int_{Q_{t}}\left(1+\theta^{r p_{n}}|\boldsymbol{\epsilon}|^{(q-1) p_{n}}\right)|\nabla \boldsymbol{\epsilon}|^{p_{n}} d x d t^{\prime} & \\
& \leq \Lambda+\Lambda \int_{Q_{t}} \theta^{2 r p_{n}}|\boldsymbol{\epsilon}|^{2(q-1) p_{n}} d x d t^{\prime}+\int_{Q_{t}}|\nabla \boldsymbol{\epsilon}|^{2 p_{n}} d x d t^{\prime} \leq \Lambda,
\end{aligned}
$$

where in the last inequality we have used the bounds (3.27), (3.34) and (3.31).

Finally, recalling (FE-1), (FE-2), we get

$$
\begin{array}{rl}
I_{2} \leq \Lambda \int_{Q_{t} \cap\left\{\theta<\theta_{1}\right\}}\left(1+|\boldsymbol{\epsilon}|^{q p_{n}}\right)|\nabla \theta|^{p_{n}} & d x d t^{\prime} \\
& +\Lambda \int_{Q_{t} \cap\left\{\theta \geq \theta_{1}\right\}} \theta^{(r-1) p_{n}}|\boldsymbol{\epsilon}|^{q p_{n}}|\nabla \theta|^{p_{n}} d x d t^{\prime} \leq \Lambda,
\end{array}
$$

where we have used the continuity of $\theta$ and the estimates (3.27), (3.34). Combining the above estimates yields the assertion.

Estimate (3.35) allows us to apply the Solonnikov theory of parabolic systems [10] to BVP (3.1) and to conclude that

$$
\mathbf{w} \in \mathbf{W}_{p_{n}}^{2,1}\left(Q_{t}\right) \quad \text { and } \quad\|\mathbf{w}\|_{\mathbf{W}_{p_{n}}^{2,1}\left(Q_{t}\right)} \leq \Lambda .
$$

Subsequently, the application of this theory to BVP (3.2) gives

$$
\mathbf{u} \in \mathbf{W}_{p_{n}}^{4,2}\left(Q_{t}\right) \quad \text { and } \quad\|\mathbf{u}\|_{\mathbf{W}_{p_{n}}^{4,2}\left(Q_{t}\right)} \leq \Lambda,
$$

and by the imbedding,

$$
\boldsymbol{\epsilon}_{t} \in \mathbf{L}_{p}\left(Q_{t}\right) \quad \text { and } \quad\left\|\boldsymbol{\epsilon}_{t}\right\|_{\mathbf{L}_{p}\left(Q_{t}\right)} \leq \Lambda \text { for } p_{n} \leq p<\infty .
$$

With this estimate we return to the temperature equation and estimate its right-hand side in $L_{p}\left(Q_{t}\right)$-norm. We obtain, for any $p_{n} \leq p<\infty$,

$$
\begin{aligned}
\int_{Q_{t}}\left|\theta F_{1 / \theta \boldsymbol{\epsilon}}(\boldsymbol{\epsilon}, \theta): \boldsymbol{\epsilon}_{t}\right|^{p} d x d t^{\prime} & \leq \frac{1}{2} \int_{Q_{t}}\left|\theta F_{1 / \theta \boldsymbol{\epsilon}}(\boldsymbol{\epsilon}, \theta)\right|^{2 p} d x d t^{\prime}+\frac{1}{2} \int_{Q_{t}}\left|\boldsymbol{\epsilon}_{t}\right|^{2 p} d x d t^{\prime} \\
& \leq \Lambda+\Lambda \int_{Q_{t}} \theta^{2 r p}|\boldsymbol{\epsilon}|^{2 q p} d x d t^{\prime}+\Lambda \int_{Q_{t}}\left|\boldsymbol{\epsilon}_{t}\right|^{2 p} d x d t^{\prime} \leq \Lambda,
\end{aligned}
$$

where we have used pointwise estimates (3.27) on $\epsilon$ and the $L_{p}$-estimates (3.34), (3.36) on $\theta$ and $\epsilon_{t}$. Hence, by recalling assumption (BV-p), the classical parabolic theory assures that

$$
\theta \in W_{p}^{2,1}\left(Q_{t}\right) \quad \text { and } \quad\|\theta\|_{W_{p}^{2,1}\left(Q_{t}\right)} \leq \Lambda \quad \text { for } p_{n} \leq p<\infty .
$$

Again, by the imbedding, it follows that $\theta$ is continuous in $Q_{t}$ and

$$
\theta \leq \Lambda \text { in } Q_{t},
$$

as well as

$$
\nabla \theta \in L_{p}\left(Q_{t}\right) \quad \text { and } \quad\|\nabla \theta\|_{L_{p}\left(Q_{t}\right)} \leq \Lambda \quad \text { for } p_{n} \leq p<\infty .
$$


In the last step, using the same arguments as in Lemma 3.6 and taking advantage of $(3.38),(3.39)$ we estimate

$$
\left\|\nabla \cdot F_{/ \boldsymbol{\epsilon}}(\boldsymbol{\epsilon}, \theta)\right\|_{\mathbf{L}_{p}\left(Q_{t}\right)} \leq \Lambda \text { for } p_{n} \leq p<\infty .
$$

Therefore, if we return to the system (3.1)-(3.3) and apply the Solonnikov theory, we obtain

$$
\|\mathbf{w}\|_{\mathbf{W}_{p}^{2,1}\left(Q_{t}\right)} \leq \Lambda \quad \text { and } \quad\|\mathbf{u}\|_{\mathbf{W}_{p}^{4,2}\left(Q_{t}\right)} \leq \Lambda \text { for } p_{n} \leq p<\infty .
$$

This completes the derivation of a priori bounds for a fixed point of the transformation $T_{1}$, meaning that the assumption (iii) of the Leray-Schauder Theorem 3.1 is satisfied.

In the last part of the proof we demonstrate assumption (i) by showing that for fixed $\tau \in[0,1], T_{\tau}$ maps bounded subsets into precompact subsets in $V(p)$.

Step 5 . Complete continuity of $T_{\tau}$ is equivalent to the following property. Let $\left(\overline{\mathbf{u}}^{n}, \bar{\theta}^{n}\right)$ be a bounded sequence in $V(p)$, such that

$$
\left(\overline{\mathbf{u}}^{n}, \bar{\theta}^{n}\right) \rightarrow(\overline{\mathbf{u}}, \bar{\theta}) \quad \text { weakly in } V(p) \text { for } n \rightarrow \infty .
$$

Then for the values of $T_{\tau}$

$$
\left(\mathbf{u}^{n}, \theta^{n}\right)=T_{\tau}\left(\overline{\mathbf{u}}^{n}, \bar{\theta}^{n}\right)
$$

we have

$$
\begin{array}{cl}
\mathbf{u}^{n} \rightarrow \mathbf{u} & \text { strongly in } \mathbf{W}_{p}^{4,2}\left(Q_{T}\right) \\
\theta^{n} \rightarrow \theta & \text { strongly in } W_{p}^{2,1}\left(Q_{T}\right)
\end{array}
$$

as $n \rightarrow \infty$, where

$$
(\mathbf{u}, \theta)=T_{\tau}(\overline{\mathbf{u}}, \bar{\theta}) .
$$

The proof of the above property, based on parabolic systems theory, is given in [9].

In this way we have established that all the assumptions of the Leray-Schauder theorem are satisfied. The proof of existence is thus finished.

4. Uniqueness proof. Here the reasoning is based on the direct comparison of two solutions by means of energy estimates and the application of Gronwall's inequality. Let $\left(\mathbf{u}^{1}, \theta^{1}\right),\left(\mathbf{u}^{2}, \theta^{2}\right) \in V(p)$ be two solutions corresponding to the same data. To simplify notation we set for $i=1,2$

$$
\begin{gathered}
\mathbf{v}=\mathbf{u}^{2}-\mathbf{u}^{1}, \eta=\theta^{2}-\theta^{1}, \boldsymbol{\epsilon}^{i}=\boldsymbol{\epsilon}\left(\mathbf{u}^{i}\right), \boldsymbol{\epsilon}_{t}^{i}=\boldsymbol{\epsilon}\left(\mathbf{u}_{t}^{i}\right), \\
F_{/ \boldsymbol{\epsilon}}^{i}=F_{/ \boldsymbol{\epsilon}}\left(\boldsymbol{\epsilon}^{i}, \theta^{i}\right), F_{/ \theta \boldsymbol{\epsilon}}^{i}=F_{/ \theta \boldsymbol{\epsilon}}\left(\boldsymbol{\epsilon}^{i}, \theta^{i}\right) .
\end{gathered}
$$

The difference $(\mathbf{v}, \eta) \in V(p)$ satisfies the BVP:

$$
\begin{gathered}
\mathbf{v}_{t t}-\nu \mathbf{Q} \mathbf{v}_{t}+\frac{\kappa}{4} \mathbf{Q Q v}=\boldsymbol{\nabla} \cdot\left(F_{/ \boldsymbol{\epsilon}}^{2}-F_{/ \boldsymbol{\epsilon}}^{1}\right), \\
c_{v} \eta_{t}-k \Delta \eta=\left(\theta^{2} F_{/ \theta \boldsymbol{\epsilon}}^{2}: \boldsymbol{\epsilon}_{t}^{2}-\theta^{1} F_{/ \theta \boldsymbol{\epsilon}}^{1}: \boldsymbol{\epsilon}_{t}^{1}\right)+\left(\nu\left(\mathbf{A} \boldsymbol{\epsilon}_{t}^{2}\right): \boldsymbol{\epsilon}_{t}^{2}-\nu\left(\mathbf{A} \boldsymbol{\epsilon}_{t}^{1}\right): \boldsymbol{\epsilon}_{t}^{1}\right) \\
\equiv R_{1}+R_{2} \quad \text { in } Q_{T}, \\
\mathbf{v}(0, x)=0, \mathbf{v}_{t}(0, x)=0, \eta(0, x)=0 \quad \text { in } \Omega, \\
\mathbf{v}=\mathbf{Q v}=0, \boldsymbol{\nabla} \eta \cdot \mathbf{n}=0 \quad \text { on } S_{T} .
\end{gathered}
$$


In the first step we obtain energy estimates for $\mathbf{v}$ in terms of $L_{2}$-norm of $\eta$. To this end we multiply (4.1) by $\mathbf{v}_{t}$ and integrate over $Q_{t}$ to get

$$
\begin{aligned}
\frac{1}{2} \int_{Q_{t}} \frac{d}{d t}\left|\mathbf{v}_{t}\right|^{2} d x d t^{\prime}-\nu \int_{Q_{t}}\left(\mathbf{Q} \mathbf{v}_{t}\right) \cdot \mathbf{v}_{t} d x d t^{\prime} & +\frac{\kappa}{4} \int_{Q_{t}}(\mathbf{Q Q v}) \cdot \mathbf{v}_{t} d x d t^{\prime} \\
& -\int_{Q_{t}}\left(\nabla \cdot\left(F_{/ \epsilon}^{2}-F_{/ \epsilon}^{1}\right)\right) \cdot \mathbf{v}_{t} d x d t^{\prime}=0
\end{aligned}
$$

Hence, integration by parts (as in the proof of Lemma 3.2) and the use of initial conditions (4.3) yield

$$
\begin{aligned}
\int_{\Omega_{t}}\left(\frac{1}{2}\left|\mathbf{v}_{t}\right|^{2}+\frac{\kappa}{8}|\mathbf{Q v}|^{2}\right) d x+\nu \int_{Q_{t}}\left(\mathbf{A} \boldsymbol{\epsilon}\left(\mathbf{v}_{t}\right)\right) & : \boldsymbol{\epsilon}\left(\mathbf{v}_{t}\right) d x d t^{\prime} \\
& =-\int_{Q_{t}}\left(F_{/ \boldsymbol{\epsilon}}^{2}-F_{/ \boldsymbol{\epsilon}}^{1}\right): \boldsymbol{\epsilon}\left(\mathbf{v}_{t}\right) d x d t^{\prime}
\end{aligned}
$$

Moreover, thanks to (4.3), we have

$$
\frac{1}{2} \int_{\Omega_{t}}|\boldsymbol{\epsilon}(\mathbf{v})|^{2} d x=\frac{1}{2} \int_{Q_{t}} \frac{d}{d t}|\boldsymbol{\epsilon}(\mathbf{v})|^{2} d x d t^{\prime}=\int_{Q_{t}} \boldsymbol{\epsilon}(\mathbf{v}): \boldsymbol{\epsilon}\left(\mathbf{v}_{t}\right) d x d t^{\prime} .
$$

Combining (4.5), (4.6), and using estimate

$$
\left|F_{/ \epsilon}^{2}-F_{/ \epsilon}^{1}\right| \leq \Lambda(|\epsilon(\mathbf{v})|+|\eta|),
$$

which follows from the regularity assumption on $F(\epsilon, \theta)$ and the uniform bounds on $\epsilon^{i}, \theta^{i}$ in $Q_{T}$, by Young's inequality we arrive at

$$
\begin{aligned}
\int_{\Omega_{t}}\left(\frac{1}{2}\left|\mathbf{v}_{t}\right|^{2}+\right. & \left.|\boldsymbol{\epsilon}(\mathbf{v})|^{2}+\frac{\kappa}{8}|\mathbf{Q} \mathbf{v}|^{2}\right) d x+a_{\star} \nu \int_{Q_{t}}\left|\boldsymbol{\epsilon}\left(\mathbf{v}_{t}\right)\right|^{2} d x d t^{\prime} \\
& \leq\left(\delta_{1}+\delta_{2}\right) \int_{Q_{t}}\left|\boldsymbol{\epsilon}\left(\mathbf{v}_{t}\right)\right|^{2} d x d t^{\prime}+\Lambda\left(\delta_{1}^{-1}+\delta_{2}^{-1}\right) \int_{Q_{t}}\left(|\boldsymbol{\epsilon}(\mathbf{v})|^{2}+|\eta|^{2}\right) d x d t^{\prime}
\end{aligned}
$$

With an appropriate choice of $\delta_{i}$ the $\boldsymbol{\epsilon}\left(\mathbf{v}_{t}\right)$-term is absorbed by the left-hand side. Subsequently, the application of Gronwall's inequality implies that

$$
\begin{aligned}
\left\|\mathbf{v}_{t}\right\|_{L_{\infty}\left(0, T ; \mathbf{L}_{2}(\Omega)\right)}+\|\boldsymbol{\epsilon}(\mathbf{v})\|_{L_{\infty}\left(0, T ; \mathbf{L}_{2}(\Omega)\right)} & \\
& +\|\mathbf{Q} \mathbf{v}\|_{L_{\infty}\left(0, T ; \mathbf{L}_{2}(\Omega)\right)}+\left\|\boldsymbol{\epsilon}\left(\mathbf{v}_{t}\right)\right\|_{\mathbf{L}_{2}\left(Q_{T}\right)} \leq \Lambda\|\eta\|_{L_{2}\left(Q_{T}\right)} .
\end{aligned}
$$

Hence, by recalling the ellipticity property of the operator $\mathbf{Q}$, it follows that

$$
\|\mathbf{v}\|_{L_{\infty}\left(0, T ; \mathbf{W}_{2}^{2}(\Omega)\right)} \leq \Lambda\|\eta\|_{L_{2}\left(Q_{T}\right)} .
$$

The energy estimates for $\eta$ follow from multiplying equation (4.2) by $\eta$ and integrating over $Q_{t}$ :

$$
\frac{c_{v}}{2} \int_{Q_{t}} \frac{d}{d t} \eta^{2} d x d t^{\prime}+k \int_{Q_{t}}|\nabla \eta|^{2} d x d t^{\prime}=\int_{Q_{t}}\left(R_{1}+R_{2}\right) \eta d x d t^{\prime} .
$$

Because of the uniform bounds on $\boldsymbol{\epsilon}^{i}, \theta^{i}, \boldsymbol{\epsilon}_{t}^{i}$ and $C^{3}$-regularity of $F_{1}(\boldsymbol{\epsilon}, \theta)$ we have

$$
\left|R_{1}\right|,\left|R_{2}\right| \leq \Lambda\left(|\eta|+|\boldsymbol{\epsilon}(\mathbf{v})|+\left|\boldsymbol{\epsilon}\left(\mathbf{v}_{t}\right)\right|\right)
$$

Hence, thanks to (4.7), the right-hand side of (4.9) is estimated by

$$
\int_{Q_{t}}\left(R_{1}+R_{2}\right) \eta d x d t^{\prime} \leq \Lambda \int_{Q_{t}} \eta^{2} d x d t^{\prime}
$$


Since $\eta(0, \mathbf{x})=0$ in $\Omega$, the application of Gronwall's inequality in (4.9) implies that $\eta=0$ in $Q_{t}$. Simultaneously, by inequality $(4.8), \mathbf{v}=0$ in $Q_{T}$. This completes the proof of uniqueness.

\section{References}

[1] M. Brokate and J. Sprekels, Hysteresis and Phase Transitions, Appl. Math. Sci. 121, Springer, New York, 1996.

[2] C. M. Dafermos and L. Hsiao, Global smooth thermomechanical processes in onedimensional nonlinear thermoviscoelasticity, Nonlinear Anal. 6 (1982), 435-454.

[3] F. FALK, Landau theory and martensitic phase transitions, Journal de Physique C4 43 (1982), 3-15.

[4] F. FAlK and P. Konopka, Three-dimensional Landau theory describing the martensitic phase transformation of shape memory alloys, Journal of Physics: Condensed Matter 2 (1990), 61-77.

[5] M. Frémond, Matériaux à mémoire de forme, C. R. Acad. Sci. Paris Sér. II Méc. Phys. Chim. Sci. Univers. Sci. Terre 304 (1987), No. 7, 239-244.

[6] O. A. Ladyzhenskaya, V. A. Solonnikov and N. N. Uralceva, Linear and Quasilinear Equations of Parabolic Type, Transl. Math. Monogr. 23, Amer. Math. Soc., Providence, 1968.

[7] J. NeČAs, Les méthodes directes en théorie des équations elliptiques, Mason, Paris, 1967.

[8] I. PAWŁow, Three-dimensional model of thermomechanical evolution of shape memory materials, Control Cybernet. 29 (2000), 341-365.

[9] I. PawŁow and A. Żochowski, Existence and uniqueness of solutions for a threedimensional thermoelastic system, Dissertationes Math. (Rozprawy Mat.) 406 (2002).

[10] V. A. Solonnikov, On the boundary value problems for the linear parabolic systems of general type (in Russian), in: Boundary Value Problems of Mathematical Physics III, O. A. Ladyzhenskaya (ed.), Trudy Mat. Inst. Steklov. 83 (1965), 3-163; English transl.: Proc. Steklov Inst. Mat. 83 (1965). 